\title{
The very fast evolution of Sakurai's object
}

\author{
G. C. Van de Steene ${ }^{1}$, P. A. M. van Hoof ${ }^{1}$, S. Kimeswenger ${ }^{2}$, \\ A. A. Zijlstra ${ }^{3}$, A. Avison ${ }^{3}$ L. Guzman-Ramirez ${ }^{4}$, M. Hajduk $^{5}$ \\ and F. Herwig ${ }^{6}$
}

\author{
${ }^{1}$ Royal Observatory of Belgium, Astronomy \& Astrophysics, Ringlaan 3, Brussels, Belgium \\ email: $g$.vandesteene@oma.be \\ ${ }^{2}$ Universidad Católica del Norte, Antofagasta, Chile \\ ${ }^{3}$ Jodrell Bank Centre for Astrophysics, Manchester, UK \\ ${ }^{4}$ Sterrewacht, Universiteit Leiden, The Netherlands \\ ${ }^{5}$ Nicolaus Copernicus Astronomical Center, Torun, Poland \\ ${ }^{6}$ University of Victoria, Victoria, Canada
}

\begin{abstract}
V4334 Sgr (a.k.a. Sakurai's object) is the central star of an old planetary nebula that underwent a very late thermal pulse a few years before its discovery in 1996. We have been monitoring the evolution of the optical emission line spectrum since 2001. The goal is to improve the evolutionary models by constraining them with the temporal evolution of the central star temperature. In addition the high resolution spectral observations obtained by X-shooter and ALMA show the temporal evolution of the different morphological components.
\end{abstract}

Keywords. planetary nebulae: individual (V4334 Sgr), evolution, Stars: AGB and post-AGB, circumstellar matter

\section{Introduction}

V4334 Sgr (a.k.a. Sakurai's object) is the central star of an old planetary nebula (PN) that underwent a very late thermal pulse (VLTP) a few years before its discovery in 1996 (Nakano et al. 1996). During the VLTP it ingested its remaining hydrogen rich envelope into the helium burning shell and ejected the processed material shortly afterwards to form a new, hydrogen deficient nebula inside the old PN. The star brightened considerably to become a very cool (born-again) AGB star with a spectrum resembling a carbon star. After a few years, dust formation started in the new ejecta and the central star became highly obscured, similar to R CrB stars. Emission lines were discovered: first He I $1083 \mathrm{~nm}$ in 1998 (Eyres et al. 1999), later in 2001 also optical forbidden lines from neutral and singly ionized nitrogen, oxygen and sulfur (Kerber et al. 2002). Sakurai baffled the scientific community with its very fast evolution, much faster than pre-discovery models predicted. The current models (Lawlor \& MacDonald 2003, Herwig et al. 2011, Herwig et al. 2014, Miller Bertolami et al. 2006) can be improved by constraining them with the temporal evolution of the central star temperature.

\section{Observations and results}

\subsection{Optical monitoring}

We have been monitoring the evolution of the optical emission line spectrum since 2001 using spectra obtained with FORS at the ESO-VLT (Fig. 1). The goal of this monitoring program is to derive the stellar temperature as a function of time (van Hoof et al. 2007, 


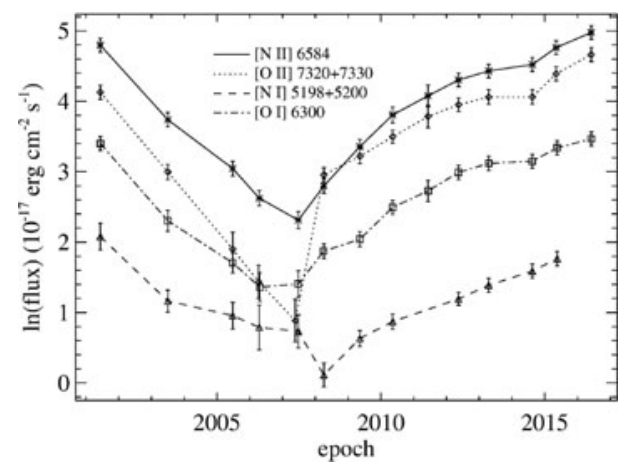

Figure 1. Evolution of emission line fluxes since 2001.

van Hoof et al. 2008). From 2001 through 2007 the optical spectrum showed an exponential decline in flux and the level of excitation also dropped. We see this as evidence for a shock that occurred around 1998 and cooled afterwards. A plausible explanation is that this is the fastest material ejected in the VLTP hitting older ejecta. The optical line fluxes started to increase again since 2008 . The sudden jump in [O II] flux in 2008 could point to a second shock as the cause of the change in behavior. The optical spectrum shows new lines which have been emerging since 2013.

Some have tentatively been identified as electronic transitions of CN. At least some of these newly emerging lines are formed very close to the central star, possibly in the disk. In the PV diagram of [N II] $658.3 \mathrm{~nm}$, obtained with X-shooter, we see that the red-and blue-shifted emission come from different regions that are spatially shifted with respect to the continuum. This emission line, as well as other optical forbidden lines, originate in the bipolar lobes seen by Hinkle \& Joyce (2014).

\subsection{ALMA Observations}

The continuum emission is unresolved which indicates that all the dust is in the disk. In the ALMA spectra we detect $\mathrm{CO}, \mathrm{CN}, \mathrm{HC}_{3} \mathrm{~N}$, as well as ${ }^{13} \mathrm{C}$ isotopologues of these species. The $\mathrm{CO}$ and $\mathrm{HC}_{3} \mathrm{~N}$ (+isotopologues) emission is unresolved, coincides with the position of the central star, and most likely comes from the disk (Fig. 2). The $\mathrm{CN}$ and ${ }^{13} \mathrm{CN}$ emission is spatially resolved and coincides with the bipolar lobes seen by Hinkle \& Joyce (2014) (Fig. 2). CN could be formed via shock-induced dissociation of $\mathrm{HCN}$ in the lobes.

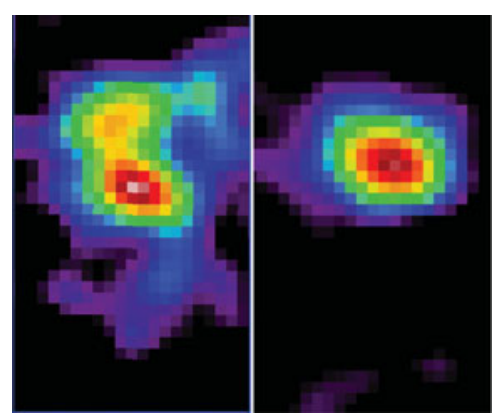

Figure 2. CN (left) and CO emission (right) detected by ALMA in 2015.

\section{References}

Chesneau, O., Clayton, G. C., Lykou, F., et al., 2009, A\&A, 493, L17

Eyres, S. P. S., Smalley, B., Geballe, T. R., et al., 1999, MNRAS, 307, L11

Hajduk, M., Zijlstra, A. A., Herwig, F., et al., 2005, Science, 308, 231

Herwig, F., Pignatari, M., Woodward, P. R., et al., 2011, ApJ, 727, 89

Herwig, F., Woodward, P. R., Lin, P.-H., Knox, M., \& Fryer, C., 2014, ApJ, 792, L3

Hinkle, K. H. \& Joyce, R. R., 2014, ApJ, 785, 146

Kerber, F., Pirzkal, N., De Marco, O., et al., 2002, ApJ, 581, L39

Lawlor, T. M. \& MacDonald, J., 2003, ApJ, 583, 913

Miller Bertolami, M. M., Althaus, L. G., Serenelli, A. M., \& Panei, J. A., 2006, A\&A, 449, 313

Nakano, S., Sakurai, Y., Hazen, M., et al., 1996, IAU Circ., 6322, 1

van Hoof, P. A. M., Hajduk, M., Zijlstra, A. A., et al., 2007, A\&SA, 471, L9

van Hoof, P. A. M., Hajduk, M., Zijlstra, A. A., et al., 2008, ASP Conf. Ser., vol. 391, 155 